\title{
Necrosis and Infiltrate
}

National Cancer Institute

\section{Source}

National Cancer Institute. Necrosis and Infiltrate. NCI Thesaurus. Code C139158.

A morphologic finding indicating the presence of necrosis associated with a cellular infiltrate in a tissue sample. 